\title{
Mental health characteristics and their associations with childhood trauma among subgroups of people living with HIV in China
}

Dongfang Wang ${ }^{1,2}$, Qijian Deng ${ }^{1}$, Brendan Ross ${ }^{3}$, Min Wang ${ }^{4}$, Zhening Liu ${ }^{1}$, Honghong Wang ${ }^{5}$ and Xuan Ouyang ${ }^{1 *}$

\begin{abstract}
Background: People living with HIV (PLWH) carry a high risk for mental health problems, which has been extensively reported in the literature. However, an understanding of mental health characteristics in different subgroups of PLWH is still limited. In the present study, we conducted a cross-sectional survey to explore mental health characteristics and their associations with childhood trauma in two major subgroups of PLWH in China.

Methods: A total of 533 PLWH (213 prisoners in the prison system, and 320 outpatients) were assessed using the 8-item Positive Subscale of the Community Assessment of Psychic Experiences (CAPE-P8), Generalized Anxiety Disorder scale (GAD-7), Patient Health Questionnaire (PHQ-9), and Childhood Trauma Questionnaire (CTQ).

Results: From the total sample, 22.0\% PLWH frequently experienced psychotic-like experiences (PLEs), 21.8\% had clinically significant anxiety syndrome, $34.0 \%$ had clinically significant depressive syndrome, and $63.6 \%$ experienced at least one type of traumatic exposure during their childhood, with physical neglect being the most common. Compared to outpatients with HIV, prisoners living with HIV reported more severe mental health problems and a higher frequency of childhood trauma, with childhood trauma in turn predicting higher risk for mental health problems. Similarly, among outpatients living with HIV, both childhood emotional and sexual abuse had predictive effects on all the three mental health problems.

Conclusions: The study suggests that PLWH have higher risk of anxiety, depression and PLEs, and childhood trauma could serve as predicting factors for such risks. In addition, childhood trauma may play distinct roles in predicting the risk for the mental health problems, depending on different subgroup of PLWH.
\end{abstract}

Keywords: People living with HIV, Psychotic-like experience, Anxiety, Depression, Childhood trauma

*Correspondence: ouyangxuan@csu.edu.cn

1 Department of Psychiatry, National Clinical Research Center on Mental Disorders, China National Technology Institute on Mental Disorders, the Second Xiangya Hospital of Central South University, Changsha 410011, China

Full list of author information is available at the end of the article

\section{Background}

A great number of studies have showed that people living with HIV (PLWH) have experienced considerable degree of psychological distress. Almost 20\% of PLWH suffer from an anxiety disorder at the clinical level [1], and $6.5 \sim 75 \%$ experienced mood or depressive disorders $[2,3]$. Additionally, there is a high clinical prevalence of HIV-related psychosis, and the incidence of psychiatric 
syndrome in patients with HIV spectrum disorders ranges from 0.2 to $15 \%$. The severity of psychiatric symptoms may increase with the progression of HIV disease [4]. It has been demonstrated that mental health status of PLWH not only significantly affects ART adherence and quality of life, but is also associated with negative experiences and behaviors, such as social isolation and stigma [5]. Notably, depressive symptoms have a particularly negative effect on adherence to ART, increasing HIVrelated morbidity and mortality [6]. Meanwhile, together with stress, depressive symptoms are more likely associated with a decreased cluster of differentiation 4 (CD4) cell count and increased viral load $[7,8]$.

People in prisons and other closed settings are defined by $\mathrm{WHO}$ as a key population group who are at increased HIV risk in all countries and regions. It is reported that HIV prevalence of people in prisons is approximately five times higher than the general population [9]. In China, with the implementation of the "Four Frees and One Care" policy [10], prisoners living with HIV are taken into centralized custody in a designated prison for standardized ART treatment. How to optimize the special disease management and how to improve the quality of life has become the focus of concern for government officers, especially prison administrators. However, related research on mental health problems among prisoners living with HIV is still limited in China.

Childhood trauma is one of the most critical risk factors for mental health problems $[11,12]$ and criminal behaviors [13]. Childhood trauma has been generally considered to be a destabilizing factor leading to the onset of psychotic symptoms and increasing biological vulnerability to the development of psychiatric disorders [14]. A growing number of researchers have identified that PLWH experience more childhood trauma than the general population [15]. Fear of violence and physical abuse has negative effects on the mental health of HIVinfected men who have sex with men (MSM) [16]. Moreover, Wang et al. have found that childhood trauma was a risk factor for recidivism among male prisoners [17]. However, the internal association between childhood trauma and mental health in prisoners living with HIV has not been fully clarified.

Psychotic-like experiences (PLEs) were also included in our study as measurable variables, with PLEs generally defined as positive symptoms of psychosis at the subclinical level [18]. PLEs have been demonstrated to be a predictive risk factor for later development of psychotic disorders [19, 20], as well as non-psychotic disorders [21]. However, PLEs among the population of PLWH, especially prisoners living with HIV, has barely been studied. In this study, we hypothesized that there would be higher rates of PLEs among the PLWH, especially among prisoners living with HIV.

To further explore these connections, we carried out a cross-sectional survey to determine prevalence of anxiety, depression and PLEs, as well as their associations with childhood trauma, among the PLWH in China. Meanwhile, we attempted to identify specific mental health characteristics among the two major subgroups of PLWH in our survey: prisoners living with HIV and outpatients living with HIV.

\section{Materials and method \\ Participants}

The sample of PLWH was recruited from Hunan Province, China, including outpatients and prisoners. Outpatients living with HIV were served by the HIV/AIDS clinic of the First Hospital of Changsha, which acts as the government appointed HIV-treatment Hospital for Hunan province. Prisoners living with HIV were recruited from two designated prison facilities for prisoners living with HIV (one was a women's prison; another was a men's prison) in Hunan.

All participants (or their guardians, if age $<18$ ) signed the informed consent. The participants who were unable to understand the questionnaires or had a history of psychiatric disorders prior to their HIV diagnosis were excluded from the study.

\section{Procedure}

Outpatients living with HIV were recruited from the patient waiting room at the HIV/AIDS clinic of the First Hospital of Changsha from March 2019 to June 2019. The outpatients completed the questionnaire by themselves and/or with the help of one research assistant. Prisoners living with HIV were recruited in a meeting room of the two chosen prisons from August 2019 to September 2019. One researcher and two prison guards accompanied them during the whole process, helping answer procedural questions. The survey was under the principle of voluntary participation, and informed consent was obtained from all participants before starting the survey. Participants could withdraw from the study at any time if they felt discomfort. We offered remuneration of $50 \mathrm{RMB}$ to participants from the clinic. For the incarcerated participants, we did not offer remuneration, but we offered free group psychotherapy services.

The survey was conducted anonymously, and all data was kept entirely confidential. The investigation was carried out in accordance with the latest version of the Declaration of Helsinki and approved by the Ethics Committees of Xiangya Nursing School of Central South University (No.2018007). Under the original agreement, the 
names of the prisons and information about the prisoners' crime were not reported here.

\section{Measures \\ PLES}

The 8-item Positive Subscale of the Community Assessment of Psychic Experiences (CAPE-P8) [22, 23] was used to assess the PLE frequency in the past month. CAPE-P8 originated from CAPE-42 [24], which addresses the following domains: delusional experiences (6 items) and hallucinatory experiences ( 2 items). In terms of frequency, responses to items range from 0 -never, 1-sometimes, 2-often, to 3-nearly always. The higher scores indicate an increased level of frequency of PLEs. The Chinese version of CAPE-P8 had adequate psychometric properties [25]. The Cronbach's alpha was 0.86 in the current sample.

\section{Anxiety}

The 7-item Generalized Anxiety Disorder scale (GAD7) [26] scale was used to measure levels of anxiety in the past two weeks. Responses to items range from 0 (not at all) to 3 (nearly every day). Higher the total scores indicate higher the levels of anxiety. The cut-off value of 10 represents identified cases of clinically anxiety, with acceptable sensitivity (89\%) and specificity (82\%). In this study, the internal consistency of the scale was high, with a Cronbach's alpha of 0.94 .

\section{Depression}

The 9-item Patient Health Questionnaire (PHQ-9) was used to assess depressive symptoms within the past two weeks [27]. Responses to items range from 0-not at all, 1- several days, 2- more than half the days, to 3- nearly every day, with higher total score showing more severe depressive symptoms. Total score $\geq 10$ had a sensitivity of $88 \%$ and a specificity of $88 \%$ for clinical level of depression. The Cronbach's alpha in this sample was 0.92 .

\section{Childhood trauma}

The 28-item Childhood Trauma Questionnaire (CTQ) was used to measure self-reported experiences of childhood trauma before 16years of age [28]. The CTQ describes five domains: emotional abuse (EA), physical abuse (PA), sexual abuse (SA), emotional neglect (EN) and physical neglect (PN). Higher CTQ scores indicate a higher level of childhood trauma experienced. Based on cut-off values of severe trauma exposure $(E A \geq 13$, $\mathrm{PA} \geq 10, \quad \mathrm{SA} \geq 8, \quad \mathrm{EN} \geq 15, \quad \mathrm{PN} \geq 10$ ), the existence of severe childhood trauma history can be identified [28]. In this sample, Cronbach's alpha for the total score was 0.77 .

\section{Statistical analysis}

Data analyses were conducted using IBM SPSS Statistics version 22.0. Descriptive statistics were performed for socio-demography, HIV-related characteristics, childhood trauma and mental health characteristics. Any CAPE-P8 item with response of 2 (often) or 3 (nearly always) was counted and referenced as frequently suffers from PLEs [29]. A cut-off score of 10 on the GAD-7 and PHQ-9 was used to respectively split the anxiety and depression groups [26, 27]. Due to the limited sample size, the data in this study did not show the expected normal distribution $(p<0.05)$. Thus, correlation analysis was conducted with Spearman's correlation coefficient to investigate associations among GAD-7, PHQ-9, CAPE-P8 and CTQ in the PLWH. The $X^{2}$ test and Independent sample $t$ test were conducted to explore the differences in socio-demography, HIVrelated characteristics, and childhood trauma of different PLWH subgroups. A series of univariate logistic regression analyses were performed to determine the associations between each independent variable (sociodemography and HIV-related characteristics) in Table 1 and mental health problems. Binary logistic regression was used to assess the associations between mental health problems (PLEs, anxiety, and depression) and childhood trauma, controlling for other socio-demographics and HIV-related characteristics. The odds ratios (OR) and 95\% confidence intervals (95\% CI) were calculated. $P$-value of less than 0.05 was considered to be statistically significant.

\section{Results}

\section{Description of the sample}

Five hundred fifty seven PLWH participated in the study. Participants with $>25 \%$ missing data were excluded $(n=24)$, leaving a total sample of 533 , of which $40.0 \%(n=213)$ were prisoners and $60 \%$ $(n=320)$ were outpatients. $90.8 \%(n=484)$ were male, and participants aged from 16.0 to 68.0 years, with a mean age of $33.01(\mathrm{SD}=9.93)$ years. Sexual behavior (53.8\%) was the most common route of HIV infection in our sample, although there were still $1 / 3$ of prisoners who suffered from HIV by sharing needles while injecting drugs. Notably, 30.4\% $(n=164)$ participants reported that they didn't know how to be infected by HIV. The average reported duration of HIV infection was 62.31 ( $\mathrm{SD}=49.68)$ months. $97.7 \%(n=521)$ participants had received ART treatment, except for only 12 outpatients living with HIV. The differences of detailed socio-demographic and HIV-related characteristics between the prisoner and outpatient group were shown in Table 1. 
Table 1 Descriptive statistics of socio-demographic and HIV-related characteristics

\begin{tabular}{|c|c|c|c|c|c|}
\hline & $\begin{array}{l}\text { Overall } \\
n=533\end{array}$ & Prisoners $n=213$ & $\begin{array}{l}\text { Outpatients } \\
n=320\end{array}$ & $x^{2} / t$ & $\mathbf{P}$ \\
\hline Age (years), M(SD) & $33.01(9.93)$ & $40.52(8.96)$ & 28.12(7.06) & 16.64 & $<.001$ \\
\hline Education (years), M(SD) & $12.41(3.82)$ & $9.02(2.75)$ & $14.65(2.58)$ & -23.38 & $<.001$ \\
\hline Duration of HIV Infection (months), M(SD) & $62.31(49.68)$ & $94.33(54.03)$ & 39.74(30.38) & 13.02 & $<.001$ \\
\hline Male, $n(\%)$ & $484(90.8)$ & $179(84.0)$ & $305(95.3)$ & 19.47 & $<.001$ \\
\hline Ethnic Han, n(\%) & 489(91.7) & 189(88.7) & 300(93.8) & 4.25 & .053 \\
\hline Residence location in rural, $\mathrm{n}(\%)$ & 203(38.1) & $89(41.8)$ & $94(29.4)$ & 18.69 & $<.001$ \\
\hline Unmarried, n(\%) & $352(66.0)$ & 83(39.0) & 269(84.1) & 127.66 & $<.001$ \\
\hline Route of infection, $n(\%)$ & & & & 111.24 & $<.001$ \\
\hline Sexuality & 287(53.8) & $80(37.6)$ & 207(64.7) & & \\
\hline Blood transfusion & $8(1.5)$ & $5(2.3)$ & $3(0.9)$ & & \\
\hline Needle sharing/ take drugs & $76(14.3)$ & $71(33.3)$ & $5(1.6)$ & & \\
\hline Unknown & 162(30.4) & $57(26.8)$ & 105(32.8) & & \\
\hline Frequent PLEs & $117(22.0)$ & $59(27.7)$ & $58(18.1)$ & 6.84 & .010 \\
\hline Depression & $181(34.0)$ & $94(44.1)$ & $87(27.2)$ & 0.12 & .784 \\
\hline Anxiety & 116(21.8) & $48(22.5)$ & $68(21.3)$ & 16.37 & $<.001$ \\
\hline
\end{tabular}

Note: Frequent PLEs was referred by any item in CAPE-P8 with response with 2 (often) or 3 (nearly always)

Depression was referred by PHQ-9 with a cut-off value of 10

Anxiety was referred by GAD-7 with a cut-off value of 10

\section{Mental health characteristics}

Table 1 also showed $22.0 \%$ of respondents $(n=117)$ reported the presence of PLEs often or nearly-always. $34.0 \%(n=181)$ had depression, and $21.8 \%(n=116)$ participants were identified as anxiety. Compared with outpatients, prisoners living with HIV exhibited more frequent PLEs $\left(27.7 \%\right.$ vs. $\left.18.1, \chi^{2}=6.84, p=.010\right)$. No significant difference in anxiety was identified between the prisoners and outpatients $\left(22.5 \%\right.$ vs. $21.3 \%, \chi^{2}=.12$, $p=.784$ ). In addition, prisoners living with HIV reported a higher proportion of depression than outpatients living with $\operatorname{HIV}\left(44.1 \%\right.$ vs. $\left.27.2 \%, \chi^{2}=16.37, p<.001\right)$. As shown in Table 2, lower education level was significantly related to poor mental health of PLWH, as well as residence location in rural as a risk factor for frequent PLEs.

\section{Childhood trauma}

Three hundred and thirty-nine (63.6\%) participants experienced at least one type of traumatic exposure during their childhood, and the most common childhood

Table 2 Univariate logistic regression of associations between mental health problems, socio-demographic and HIV-related characteristics [Crude OR (95\% Cl)]

\begin{tabular}{llll}
\hline & Frequent PLEs $\mathbf{n = 1 1 7}$ & $\begin{array}{c}\text { Anxiety } \\
\mathbf{n = 1 1 6}\end{array}$ & $\begin{array}{c}\text { Depression } \\
\mathbf{n = 1 8 1}\end{array}$ \\
\hline Age (years) & $1.02(0.99,1.04)$ & $1.02(0.99,1.04)$ & $1.03(1.01,1.05)^{* *}$ \\
Education (years) & $0.87(0.82,0.92)^{* * *}$ & $0.95(0.89,0.99)^{*}$ & $0.91(0.87,0.96)^{* * *}$ \\
Duration of HIV Infection (months) & $1.00(1.00,1.01)$ & $1.00(1.00,1.01)$ & $1.01(1.00,1.01)$ \\
Male, (Female as Ref.) & $2.13(0.89,5.14)$ & $2.11(0.87,5.08)$ & $1.47(0.76,2.85)$ \\
Ethnic Han, (Others as Ref.) & $1.53(0.67,3.54)$ & $1.84(0.76,4.46)$ & $1.60(0.79,3.24)$ \\
Residence location in rural, (Urban as Ref.) & $1.76(1.12,2.75)^{*}$ & $1.41(0.91,2.19)$ & $1.33(0.92,1.94)$ \\
Unmarried, (Married as Ref.) & $0.82(0.83,1.25)$ & $1.13(0.73,1.75)$ & $0.91(0.62,1.33)$ \\
Route of infection, (Sexuality as Ref.) & & & $1.29(0.25,6.55)$ \\
Blood transfusion & $1.29(0.25,6.55)$ & $1.48(0.83,2.63)$ & $1.31(0.31,5.62)$ \\
Needle sharing/ take drugs & $1.48(0.83,2.63)$ & $1.03(0.64,1.65)$ & $1.68(1.00,2.82)$ \\
Unknown & $1.07(0.67,1.71)$ & $1.13(0.75,1.70)$ \\
\hline
\end{tabular}

Note: OR: Odd-Ratio; Cl: Confidence interval; ${ }^{*} p<.05 ;{ }^{* *} p<.01 ;{ }^{* * *} \mathrm{p}<.001$ 
trauma was PN (49.9\%). Compared to outpatients living with HIV, prisoners living with HIV had higher proportions of exposure to childhood trauma, except for sexual abuse $(p>.05)$ (see Table 3).

\section{Correlation analysis}

CTQ total score showed significant positive correlations with the total scores of CAPE-P8 $(\mathrm{r}=0.34, p<.001)$, GAD-7 $\quad(\mathrm{r}=0.25, \quad p<0.001)$, and PHQ-9 $\quad(\mathrm{r}=0.31$, $p<.001)$.

\section{Predicting effects of childhood trauma on mental health characteristics}

Odds ratios (OR) and confidence intervals (CI) for each potential predicting variable were listed in Table 4 . Three types of traumatic exposure were identified as significant predictors among prisoners living with HIV, including $\mathrm{PN}$ on PLEs $(\mathrm{OR}=3.19, \mathrm{CI}=1.09-9.35)$, EA on anxiety $(\mathrm{OR}=7.77, \mathrm{CI}=1.78-33.84), \mathrm{SA}$ on anxiety $(\mathrm{OR}=2.50, \mathrm{CI}=1.01-6.23)$ and depression

Table 3 Childhood trauma in PLWHs [n(\%)]

\begin{tabular}{llllll}
\hline & $\begin{array}{l}\text { Overall } \\
\mathbf{n}=\mathbf{5 3 3}\end{array}$ & $\begin{array}{l}\text { Prisoners } \\
\mathbf{n}=\mathbf{2 1 3}\end{array}$ & $\begin{array}{l}\text { Outpatients } \\
\mathbf{n}=\mathbf{3 2 0}\end{array}$ & $\mathbf{x}^{\mathbf{2}}$ & $\mathbf{p}$ \\
\hline EA & $32(6.0)$ & $21(9.9)$ & $11(3.4)$ & 9.35 & 0.003 \\
PA & $58(10.9)$ & $41(19.2)$ & $17(5.3)$ & 25.61 & $<0.001$ \\
SA & $137(25.7)$ & $57(26.8)$ & $80(25.0)$ & 0.21 & 0.69 \\
EN & $192(36.0)$ & $103(48.4)$ & $89(27.8)$ & 23.42 & $<0.001$ \\
PN & $266(49.9)$ & $145(68.1)$ & $121(37.8)$ & 46.85 & $<0.001$ \\
Any type & $339(63.6)$ & $160(75.1)$ & $179(55.9)$ & 20.32 & $<0.001$ \\
of trauma & & & & & \\
\hline
\end{tabular}

Note: $E A$ emotional abuse, $P A$ physical abuse, $S A$ sexual abuse, $E N$ emotional neglect, $P N$ physical neglect
$(\mathrm{OR}=2.22, \mathrm{CI}=1.02-4.87)$. For outpatients living with HIV, EA and SA showed significantly positive effects on PLEs (EA: $\mathrm{OR}=5.94, \mathrm{CI}=1.37-25.72 ; \mathrm{SA}: \mathrm{OR}=2.82$, $\mathrm{CI}=1.31-6.09)$, anxiety $(\mathrm{EA}: \mathrm{OR}=5.36, \mathrm{CI}=1.31-21.84$; $\mathrm{SA}: \mathrm{OR}=2.96, \mathrm{CI}=1.46-6.00$ ), and depression (EA: $\mathrm{OR}=8.64, \mathrm{CI}=1.83-40.79$; $\mathrm{SA}: \mathrm{OR}=2.50, \mathrm{CI}=1.29$ 4.82), while PA, EN, and PN lacked significant predictive effects on the mental health of this sample.

\section{Discussion}

Our study examined mental health characteristics (anxiety, depression and PLEs) and their associations with childhood trauma among two major subgroups of PLWH (prisoners and outpatients). It is the first pilot study to explore PLEs among PLWH, as well as the differences in mental health characteristics and factors of childhood trauma between the two subgroups of PLWH.

In our study, PLWH reported a high proportion of mental health problems, with $21.8 \%$ identified as high risk for anxiety and $34.0 \%$ identified as high risk for depression. This finding is consistent with previous studies. Based on the PHQ-9 and GAD-7 with a cut-off score of 10, data from across PLWH with ART treatment in U.S. also indicated elevated rates of depression (30\%) [30]. In study of 667 PLWH (92\% male) in China, 34.5\% presented with moderate or severe depression, $27.3 \%$ presented with moderate or severe anxiety [31].

In addition, 22.0\% PLWH experienced frequent PLEs in the current sample. Although PLEs are regarded as an important endophenotype in psychopathology, there is still lack of concern of their presence in PLWH. Notably, prisoners living with HIV showed a higher risk for PLEs and depression compared to outpatients in the study. These findings suggest more robust psychosocial

Table 4 Results of the logistic regression in $\mathrm{PLWHs}^{\mathrm{a}}$

\begin{tabular}{|c|c|c|c|c|c|c|c|}
\hline & & \multicolumn{2}{|c|}{ Frequent PLEs } & \multicolumn{2}{|l|}{ Anxiety } & \multirow[b]{2}{*}{ OR } & \multirow{2}{*}{$\begin{array}{l}\text { Depression } \\
\mathrm{Cl}(95 \%)\end{array}$} \\
\hline & & OR & $\mathrm{Cl}(95 \%)$ & OR & $\mathrm{Cl}(95 \%)$ & & \\
\hline \multirow[t]{5}{*}{ Prisoners } & EA & $2.10(.277)$ & $0.55,8.04$ & 7.77(.006) & $1.78,33.84$ & $4.01(.070)$ & $0.89,18.06$ \\
\hline & PA & $1.08(.881)$ & $0.38,3.08$ & $1.46(.511)$ & $0.47,4.51$ & $1.49(.422)$ & $0.56,3.98$ \\
\hline & SA & $1.97(.107)$ & $0.86,4.50$ & $2.50(.048)$ & $1.01,6.23$ & $2.22(.046)$ & $1.02,4.87$ \\
\hline & EN & $1.52(.319)$ & $0.67,3.47$ & $0.64(.370)$ & $0.25,1.68$ & $0.76(.459)$ & $0.36,1.59$ \\
\hline & PN & $3.19(.034)$ & $1.09,9.35$ & $3.47(.052)$ & $0.99,12.16$ & $1.24(.590)$ & $0.57,2.72$ \\
\hline \multirow[t]{5}{*}{ Outpatients } & EA & $5.94(.017)$ & $1.37,25.72$ & $5.36(.019)$ & $1.31,21.84$ & $8.64(.006)$ & $1.83,40.79$ \\
\hline & PA & $0.83(.803)$ & $0.19,3.57$ & $1.41(.616)$ & $0.37,5.33$ & $0.36(.157)$ & $0.08,1.49$ \\
\hline & SA & $2.82(.008)$ & $1.31,6.09$ & $2.96(.003)$ & $1.46,6.00$ & $2.50(.006)$ & $1.29,4.82$ \\
\hline & EN & $2.24(.067)$ & $0.94,5.32$ & $1.67(.231)$ & $0.72,3.89$ & $1.84(.120)$ & $0.85,3.96$ \\
\hline & PN & $0.70(.410)$ & $0.30,1.63$ & $0.57(.189)$ & $0.25,1.32$ & $0.88(.727)$ & $0.42,1.84$ \\
\hline
\end{tabular}

Note: a adjusting for other socio-demographics and HIV-related characteristics 
interventions should be offered to this subgroup of PLWH because of their long-term incarceration and lack of family and societal support.

Adverse childhood experiences have been demonstrated to be common among populations of PLWH [15]. In our study, 63.6\% PLWH experienced childhood trauma in the study, with physical neglect as the most common type (49.9\%). Childhood trauma has been recognized as a significant predictor of HIV infection risk [32]. A cross-sectional study in South Africa showed those with childhood trauma were more likely to be at risk of HIV infection [33], which has been further demonstrated in another longitudinal study in rural South African youth [34]. One possible reason for this phenomenon is that people with childhood trauma are more likely to participate in a range of sexually risky behaviors [35], including unprotected sex and increased number of sex partners [36]. Another possible reason is that childhood trauma is closely related to drug use [37]. In our study, prisoners living with HIV reported more childhood trauma than outpatients. Previous studies also purported that adverse childhood experiences are critical risk factors for criminal behavior [38], with physical and sexual abuse increasing juvenile delinquency and adult criminality [39]. As previous studies have reported [40], we also found more childhood PN in prisoners living with HIV than outpatients. Lower levels of education and unhealthy lifestyles (e.g. drug abuse) may partly contribute to a higher likelihood of suffering from PN [41].

In line with previous studies [22], childhood trauma was found to be significantly and positively associated with PLEs, depression and anxiety in PLWH in this study. A strong body of evidence suggests that childhood trauma not only has a positive relationship with a broad range of mental health problems in the general population [42], but also exerts a significant and long-lasting impact on psychiatric disorders, worse quality of life and poor medication adherence, faster disease progression, and greater mortality rates among PLWH [43].

Furthermore, we explored the effects of five types of childhood trauma (EA, PA, SA, EN, PN) on mental health in different subgroup of PLWH, controlling for other socio-demographic and HIV-related characteristics. Our findings suggest that both EA and SA are risk factors predictive of PLEs, anxiety, and depression in outpatients living with HIV, as well as for anxiety in prisoners living with HIV. Prior studies have indicated that both EA and SA exhibit a stronger relationship with PLEs rather than other types of childhood trauma [44]. It has been suggested that childhood EA may decrease the ability of maladaptive cognitive emotion regulation, influencing current depression or anxiety severity [45]. Also, one study found that SA was closely associated with a lifetime diagnosis of anxiety disorder and major depression [46]. Notably, PN (i.e. the most common form of childhood trauma) was found to have a predictive effect only on PLEs in prisoners living with HIV, while there was no such effect on outpatients in our study. We speculate that childhood PN implies an adverse childhood environment (e.g. not have enough to eat), which interacts with genetic factors, resulting in a persistence of PLEs, and ultimately developing into poor outcomes [19].

Our findings indicated that childhood trauma plays an important role in PLWH's mental health. PLWH who have experienced trauma or suffered from mental disorders face unique challenges in their HIV care engagement and ARV adherence [5, 47]. Starting from trauma or mental health problems may be an effective way to protect the health of HIV patients clinically. For example, improving AIDS Care after Trauma (ImpACT) is effective in reducing Post-traumatic stress disorder (PTSD) symptoms and increasing ART adherence motivation among PLWH [48]. Meanwhile, cognitive-behavioural therapy for adherence and depression (CBT-AD) has the potential to improve clinical depression, ART adherence and viral load for virally unsuppressed PLWH [49].

Some limitations should be considered for the study. There may be potential reporting bias, because detection of mental health problems relied on self-report questionnaires rather than clinical interviews in this study. In the future, clinical diagnosis should be involved to determine the prevalence rate of mental disorders. Meanwhile, the cut off score of 10 for GAD-7 and PHQ-9 are widely recognized among the general population. However, when it comes to the population of PLWH, such criterion should be treated with caution. For example, it has been demonstrated that PHQ-9 has high specificity but apparently low sensitivity for detecting MDD in HIV patients in low-income countries [50]. Thus, it remains necessary for further research to determine the performance of proven screening tools in the population of PLWH. Moreover, as for the prisoners: in terms of their management, all of the HIV-positive patients are imprisoned together, regardless of which law they may have broken, and thus we have no way of knowing when exactly they were infected. At the same time, for the clinic-based participants, it is difficult to know the timing of infection for a large majority of patients, unless it is due to certain circumstances (such as donating blood). Therefore, nature of prison sentences or length of stay and timing of infection this factor is difficult to accurately collect in our study. In addition, some other factors also may cause bias in our results, including significant differences in socio-demographic and HIV-related factors between the two subgroups, missing data, and impact of the life event of imprisonment for prisoners. 


\section{Conclusion}

In summary, this is the first exploratory study evaluating PLEs, depression and anxiety, as well as the predictive effects of childhood trauma on these mental health problems among the subgroups of PLWH in China. Importantly, our findings suggest that there are more severe mental health problems (PLEs and depression) and childhood trauma among prisoners living with HIV. Childhood emotional abuse and sexual abuse are strong predictive factors for PLEs, anxiety, and depression in PLWH. In particular, childhood physical neglect is a specific risk factor for PLEs among prisoners living with HIV. These findings support the importance of early intervention for childhood trauma, which may decrease occurring rates of PLEs, depression and anxiety, and even HIV infection.

\section{Acknowledgements}

The authors would like to show their great gratitude to all participants and related workers in this study for their participation.

\section{Authors' contributions}

DW contributed significantly to acquisition and interpretation of data, drafting and revising of the manuscript for publication. QD and BR was significantly involved in the conception, design, and analysis of the data. MW, ZL, and HW contributed to the drafting and revision of the manuscript and gave final approval to its publication. XOY was significantly involved in the conception and design of the study as well as the revision of the manuscript and final approval of its publication. All authors read and approved the final manuscript.

\section{Funding}

This work was supported by the China Precision Medicine Initiative (2016YFC0906300); the National Natural Science Foundation of China (81671335, 81701325, 81801353, 82071506); and Fogarty Center Project of NIH (D43 TW 009579).

\section{Availability of data and materials}

The datasets used and/or analyzed during the current study are available from the corresponding author (Xuan Ouyang: ouyangxuan@csu.edu.cn) on reasonable request.

\section{Declarations}

\section{Ethics approval and consent to participate}

The survey was carried out in accordance with the latest version of the Declaration of Helsinki and approved by the Ethics Committees of Xiangya Nursing School of Central South University (No.2018007). The survey was under the principle of voluntary participation. The participants (or their guardians, if age $<18$ ) carefully read, signed and returned the informed consent form to the researcher. The participants were assured that the information obtained would remain confidential.

\section{Consent for publication}

Not applicable.

\section{Competing interests}

We declare that there are no competing interests, financial or non-financial.

\section{Author details}

${ }^{1}$ Department of Psychiatry, National Clinical Research Center on Mental Disorders, China National Technology Institute on Mental Disorders, the Second Xiangya Hospital of Central South University, Changsha 410011, China. ${ }^{2}$ School of Psychology, Centre for Studies of Psychological Applications, Guangdong Key Laboratory of Mental Health and Cognitive Science, Ministry of Education Key Laboratory of Brain Cognition and Educational Science,
South China Normal University, Guangzhou, China. ${ }^{3}$ Faculty of Medicine, McGill University, Montreal, QC, Canada. ${ }^{4}$ Institute for HIV/AIDS, the First Hospital of Changsha, Changsha, China. ${ }^{5}$ Xiangya Nursing School, Central South University, Changsha, China.

Received: 24 May 2021 Accepted: 14 December 2021

Published online: 05 January 2022

\section{References}

1. Vitiello B, Burnam MA, Bing EG, Beckman R, Shapiro MF. Use of psychotropic medications among HIV-infected patients in the United States. Am J Psychiatry. 2003;160(3):547-54.

2. Gold JA, Grill M, Peterson J, Pilcher C, Lee E, Hecht FM, et al. Longitudinal characterization of depression and mood states beginning in primary HIV infection. AIDS Behav. 2014;18(6):1124-32.

3. Kagee A, Nel A, Saal W. Factor structure of the Beck depression inventoryIl among south Africans receiving antiretroviral therapy. AIDS Care. 2014:26(2):257-62.

4. Sewell DD. Schizophrenia and HIV. Schizophr Bull. 1996;22(3):465-73.

5. Nanni MG, Caruso R, Mitchell AJ, Meggiolaro E, Grassi L. Depression in HIV infected patients: a review. Curr Psychiatry Rep. 2015;17(1):530.

6. Gonzalez JS, Batchelder AW, Psaros C, Safren SA. Depression and HIV/AIDS treatment nonadherence: a review and meta-analysis. J Acquir Immune Defic Syndr. 2011;58(2):181-7.

7. Ironson G, O'Cleirigh C, Fletcher MA, Laurenceau JP, Balbin E, Klimas N, et al. Psychosocial factors predict CD4 and viral load change in men and women with human immunodeficiency virus in the era of highly active antiretroviral treatment. Psychosom Med. 2005;67(6):1013-21.

8. Leserman J, Petitto JM, Perkins DO, Folds JD, Golden RN, Evans DL. Severe stress, depressive symptoms, and changes in lymphocyte subsets in human immunodeficiency virus-infected men. A 2-year follow-up study. Arch Gen Psychiatry. 1997;54(3):279-85.

9. Maruschak LM. Bureau of Justice Statistics Bulletin: HIV in prisons, 2001-2010. (NCJ238877). Available online at: https://bjs.ojp.gov/content/ pub/pdf/hivp10.pdf.

10. Ministry of Health People's Republic China. China AIDS response Progress report 2012. China: Beijing; 2012.

11. Jonas S, Bebbington P, McManus S, Meltzer H, Jenkins R, Kuipers E, et al. Sexual abuse and psychiatric disorder in England: results from the 2007 adult psychiatric morbidity survey. Psychol Med. 2011;41(4):709-19.

12. Springer KW, Sheridan J, Kuo D, Carnes M. Long-term physical and mental health consequences of childhood physical abuse: results from a large population-based sample of men and women. Child Abuse Negl. 2007:31(5):517-30.

13. Widom CS. National Institute of Justice: An update on the "cycle of violence". Available online https://www.ojp.gov/pdffiles1/nij/184894.pdf.

14. Lambert HK, Meza R, Martin P, Fearey E, Mclaughlin KA. Childhood trauma as a public health issue: Springer International Publishing; 2017.

15. Whetten K, Reif S, Whetten R, Murphy-McMillan LK. Trauma, mental health, distrust, and stigma among HIV-positive persons: implications for effective care. Psychosom Med. 2008;70(5):531-8.

16. Phillips GN, Hightow-Weidman LB, Fields SD, Giordano TP, Outlaw AY, Halpern-Felsher B, et al. Experiences of community and parental violence among HIV-positive young racial/ethnic minority men who have sex with men. AIDS Care. 2014;26(7):827-34.

17. Wang W, Wu R, Tang H, Wang Y, Liu K, Liu C, et al. Childhood trauma as a mediator between emotional intelligence and recidivism in male offenders. Child Abuse Negl. 2019;93:162-9.

18. Lee KW, Chan KW, Chang WC, Lee EH, Hui CL, Chen EY. A systematic review on definitions and assessments of psychotic-like experiences. Early Interv Psychia. 2016;10(1):3-16.

19. Van Os J, Linscott RJ, Myingermeys I, Delespaul P, Krabbendam L. A systematic review and meta-analysis of the psychosis continuum: evidence for a psychosis proneness-persistence-impairment model of psychotic disorder. Psychol Med. 2009;39(2):179-95.

20. Dominguez MDG, Wichers M, Lieb R, Wittchen HU, Os JV. Evidence that onset of clinical psychosis is an outcome of progressively more persistent subclinical psychotic experiences: an 8-year cohort study. Schizophr Bull. 2011;37(1):84-93. 
21. Kirli U, Binbay T, Drukker M, Elbi H, Kayahan B, Keskin GD, et al. DSM outcomes of psychotic experiences and associated risk factors: 6-year follow-up study in a community-based sample. Psychol Med. 2019;49(8):1346-56.

22. Arseneault L, Cannon M, Fisher HL, Polanczyk G, Moffitt TE, Caspi A. Childhood trauma and children's emerging psychotic symptoms: a genetically sensitive longitudinal cohort study. Am J Psychiatry. 2011;168(1):65-72.

23. McGrath JJ, Saha S, Al-Hamzawi A, Alonso J, Bromet EJ, Bruffaerts R, et al. Psychotic experiences in the general population: a cross-national analysis based on 31,261 respondents from 18 countries. JAMA Psychiatry. 2015;72(7):697-705.

24. Stefanis NC, Hanssen M, Smirnis NK, Avramopoulos DA, Evdokimidis IK, Stefanis CN, et al. Evidence that three dimensions of psychosis have a distribution in the general population. Psychol Med. 2002;32(2):347-58.

25. Wang D, Sun M, Xi C, Yang XH, Mwansisya TE, Ouyang X, et al. Gender and longitudinal measurement invariance of the community assessment of psychic experiences-positive subscale. Chin J Clini Psych. 2020;01(28):41-5.

26. Spitzer RL, Kroenke K, Williams JBW, Lwe B. A brief measure for assessing generalized anxiety disorder: the GAD-7. Arch Intern Med. 2006;166(10):1092-7.

27. Kroenke K, Spitzer RL, Williams JB. The PHQ-9: validity of a brief depression severity measure. J Gen Intern Med. 2001;16(9):606-13.

28. Bernstein DP, Fink L. Childhood trauma questionnaire, a retrospective selfreport manual. San Antonio, TX: The Psychological Corporation; 1998.

29. Sun M, Zhang W, Guo R, Hu A, Li Y, Mwansisya TE, et al. Psychotic-like experiences and correlation with childhood trauma and other sociodemographic factors: a cross-sectional survey in adolescence and early adulthood in China. Psychiatry Res. 2017;255:272-7.

30. Eaton EF, Gravett RM, Tamhane AR, Mugavero MJ. Antiretroviral therapy initiation and changes in self-reported depression. Clin Infect Dis. 2017;64(12):1791-4.

31. Niu L, Qiu Y, Luo D, Chen X, Wang M, Pakenham Kl, et al. Cross-culture validation of the HIV/AIDS stress scale: the development of a revised Chinese version. PLoS One. 2016;11(4):e0152990.

32. Brown DW, Anda RF. Adverse childhood experiences: origins of behaviors that sustain the HIV epidemic. AIDS. 2009;23(16):2231-3.

33. Gibbs A, Dunkle K, Washington L, Willan S, Shai N, Jewkes R. Childhood traumas as a risk factor for HIV-risk behaviours amongst young women and men living in urban informal settlements in South Africa: a crosssectional study. PLoS One. 2018;13(4):e0195369.

34. Jewkes RK, Dunkle K, Nduna M, Jama PN, Puren A. Associations between childhood adversity and depression, substance abuse and HIV and HSV2 incident infections in rural south African youth. Child Abuse Negl. 2010;34(11):833-41.

35. Norman RE, Byambaa M, De R, Butchart A, Scott J, Vos T. The longterm health consequences of child physical abuse, emotional abuse, and neglect: a systematic review and meta-analysis. PLoS Med. 2012;9(11):e1001349.

36. Arriola KR, Louden T, Doldren MA, Fortenberry RM. A meta-analysis of the relationship of child sexual abuse to HIV risk behavior among women. Child Abuse Negl. 2005;29(6):725-46.

37. Huang C, Yuan Q, Zhang L, Wang L, Cui S, Zhang K, et al. Associations between childhood trauma and the age of first-time drug use in methamphetamine-dependent patients. Front Psychiatry. 2021;12:658205.

38. Brewer-Smyth K, Cornelius ME, Pickelsimer EE. Childhood adversity, mental health, and violent crime. J Forensic Nurs. 2015;11(1):4-14.

39. Trickett PK, Noll JG, Putnam FW. The impact of sexual abuse on female development: lessons from a multigenerational, longitudinal research study. Dev Psychopathol. 2011;23(2):453-76.

40. Li X, Wang Z, Hou Y, Wang Y, Liu J, Wang C. Effects of childhood trauma on personality in a sample of Chinese adolescents. Child Abuse Negl. 2014;38(4):788-96.

41. Carter V, Myers MR. Exploring the risks of substantiated physical neglect related to poverty and parental characteristics: a national sample. Children Youth Serv Rev. 2007:29(1):110-21.

42. Rafiq S, Campodonico C, Varese F. The relationship between childhood adversities and dissociation in severe mental illness: a meta-analytic review. Acta Psychiatr Scand. 2018;138(6):509-25.

43. Bekele T, Collins EJ, Maunder RG, Gardner S, Rueda S, Globerman J, et al. Childhood adversities and physical and mental health outcomes in adults living with HIV: findings from the Ontario HIV treatment network cohort study. AIDS Res Treat. 2018;2018:2187232.

44. Lu D, Wang W, Qiu X, Qing Z, Lin X, Liu F, et al. The prevalence of confirmed childhood trauma and its'impact on psychotic-like experiences in a sample of Chinese adolescents. Psychiatry Res. 2020;287:112897.

45. Huh HJ, Kim KH, Lee HK, Chae JH. The relationship between childhood trauma and the severity of adulthood depression and anxiety symptoms in a clinical sample: the mediating role of cognitive emotion regulation strategies. J Affect Disord. 2017:213:44-50.

46. Chen LP, Murad MH, Paras ML, Colbenson KM, Sattler AL, Goranson EN, et al. Sexual abuse and lifetime diagnosis of psychiatric disorders: systematic review and meta-analysis. Mayo Clin Proc. 2010;85(7):618-29.

47. Knettel BA, Mulawa MI, Knippler ET, Ciya N, Robertson C, Joska JA, et al. Women's perspectives on ImpACT: a coping intervention to address sexual trauma and improve HIV care engagement in Cape Town. South Africa AIDS Care. 2019:31(11):1389-96.

48. Sikkema KJ, Mulawa MI, Robertson C, Watt MH, Ciya N, Stein DJ, et al. Improving AIDS care after trauma (ImpACT): pilot outcomes of a coping intervention among HIV-infected women with sexual trauma in South Africa. AIDS Behav. 2018:22(3):1039-52.

49. Safren SA, O'Cleirigh C, Andersen LS, Magidson JF, Lee JS, Bainter SA, et al. Treating depression and improving adherence in HIV care with task-shared cognitive behavioural therapy in Khayelitsha, South Africa: a randomized controlled trial. J Int AIDS Soc. 2021;24(10):e25823.

50. Pence BW, Gaynes BN, Atashili J, O’Donnell JK, Tayong G, Kats D, et al. Validity of an interviewer-administered patient health questionnaire- 9 to screen for depression in HIV-infected patients in Cameroon. J Affect Disord. 2012;143(1-3):208-13.

\section{Publisher's Note}

Springer Nature remains neutral with regard to jurisdictional claims in published maps and institutional affiliations.
Ready to submit your research? Choose BMC and benefit from:

- fast, convenient online submission

- thorough peer review by experienced researchers in your field

- rapid publication on acceptance

- support for research data, including large and complex data types

- gold Open Access which fosters wider collaboration and increased citations

- maximum visibility for your research: over 100M website views per year

At BMC, research is always in progress.

Learn more biomedcentral.com/submissions 